\title{
Risk factors and prognostic significance of altered left ventricular geometry in preterm infants
}

\author{
Swati Choudhry ${ }^{1} \cdot$ Amber Salter $^{2} \cdot$ Tyler W. Cunningham $^{1} \cdot$ Philip T. Levy $^{1,3} \cdot$ Brian P. Hackett $^{1} \cdot$ Gautam K. Singh $^{1} \cdot$ \\ Mark C. Johnson ${ }^{1}$
}

Received: 25 June 2017 / Accepted: 23 November 2017 / Published online: 6 February 2018

(c) Nature America, Inc., part of Springer Nature 2018

\begin{abstract}
Objective: Left ventricular (LV) hypertrophy (LVH) predicts adverse cardiac events in adults. We sought to determine the risk factors and prognostic significance of altered LV geometry in preterm infants.

Study design: In an echocardiographic, single-center, retrospective case-control study we investigated the risk factors and outcomes in patients with altered LV geometry (either increased left ventricular mass index (LVMI) or increased relative wall thickness (RWT)) from a cohort of 503 preterm infants $\leq 2 \mathrm{~kg}$.

Result: Altered LV geometry was seen in 180 patients and was predicted by postnatal steroids and small for gestational age. Hospital stay was longer in the elevated RWT cases. Altered LV geometry resolved in 129 of the 131 cases with follow-up echocardiogram. Fifteen of 94 patients with elevated RWT died compared to 3/90 controls $(P=0.004)$.

Conclusion: Altered LV geometry in preterm infants is associated with postnatal steroid use and small for gestational age. Elevated RWT is associated with longer hospital stay and increased mortality.
\end{abstract}

\section{Introduction}

Left ventricular (LV) hypertrophy (LVH), as defined by elevated left ventricular mass (LVM), is an important predictor of adverse cardiovascular outcomes in adults [1,2]. The link between outcomes and LVH in children is presumed based on pediatric studies that demonstrate LVH is associated with hypertension and obesity [3, 4]. Therefore, increased attention is being paid to the timely identification of increased indexed LVM during childhood to determine if LVH can be tracked as a useful marker of cardiovascular risk $[5,6]$. Adult studies have utilized LVM in combination with relative wall thickness (RWT) to define four patterns of LV geometry. These include normal (indexed LVM and

Mark C. Johnson

Johnson_M@wustl.edu

1 Department of Pediatrics, Washington University School of Medicine, Saint Louis, MO, USA

2 Department of Biostatistics, Washington University School of Medicine, Saint Louis, MO, USA

3 Department of Pediatrics, Goryeb Children's Hospital, Morristown, NJ, USA
RWT are normal), concentric remodeling (CR, elevated RWT with normal indexed LVM), concentric hypertrophy (CH, indexed LVM and RWT are both elevated) and eccentric hypertrophy (EH, elevated indexed LVM with normal RWT) [7, 8]. The pattern of concentric hypertrophy is associated with the highest prevalence of mortality in adults [7-9].

Even though LVH predicts cardiovascular events in adults, scarce data on the prognostic impact of this problem are available in the preterm population. Aye et al. [10] found increased LVM in preterm infants that developed after birth but did not investigate the impact of LVH on outcome. RWT has not been previously used as a tool to analyze cardiac pathology in preterm infants. Previous studies have identified steroid use in preterm infants, maternal diabetes, elevated blood pressure, and intrauterine growth restriction as risk factors for LVH [11-14]. Early recognition of risk factors may help discern strategies to prevent this complication in this vulnerable population. Diagnosis of LVH has been challenging in preterm infants due to a lack of normative LV morphometric values [15]. Our previous work utilizing M-mode echocardiography found that centile curves of LVM indexed to weight are a practical method to assess LV morphology in preterm infants $\leq 2 \mathrm{~kg}$, but validation of these echocardiographic 
reference values with clinical outcomes study has not been performed [16]. Thus, the objectives of this retrospective case-control study were to determine the prevalence, risk factors, and prognostic significance of the various patterns of LV geometry in a community of hospitalized preterm infants $\leq 2 \mathrm{~kg}$.

\section{Patients and methods}

\section{Study design and participants}

For the purpose of this retrospective study, LV M-mode echocardiographic remeasurements were made in two cohorts of preterm infants: (1) a prospective cohort of 131 preterm infants (born less than 29 weeks of gestational age) was recruited between August 2011 and November 2013, and (2) a retrospective database-generated cohort of 372 preterm infants from 1 January 2005 through 31 December 2014. The institutional review board of Washington University School of Medicine approved the study. All subject guardians in the prospective sample provided written informed consent.

\section{Retrospective study population}

The last 10-year echocardiographic and clinical databases for St. Louis Children's Hospital were retrospectively reviewed. All preterm infants $\leq 2 \mathrm{~kg}$ born from 2005 to 2014 with a technically adequate echocardiographic evaluation (defined as an echocardiogram with measurable $\mathrm{M}$ mode) performed at St. Louis Children's Hospital were eligible for inclusion. Exclusion criteria were: (1) congenital heart disease; (2) known genetic cardiomyopathy including hypertrophic cardiomyopathy, genetic syndromes (such as Noonan, Pompe's disease), neuromuscular disease, chromosomal abnormalities, diagnosis of pulmonary hypertension (diagnosed based on clinical chart review or echocardiographic interpretation), connective tissue disease, and clinical or radiologic diagnosis of kidney disease; (3) patients with incomplete medical records; and (4) enrollment in the prospective sample (described below).

\section{Prospective study population}

Additionally, 131 preterm infants were prospectively enrolled from among infants participating in the Prematurity and Respiratory Outcomes Program (PROP), a 7-center initiative sponsored by the National Heart, Lung and Blood Institute (Clinical Trials number: NCT01435187) [17]. All infants in the prospective sample were enrolled at St. Louis Children's Hospital neonatal intensive care unit between August 2011 and November 2013. All prospective subjects had structurally normal hearts; none had a family history of genetic cardiomyopathy, genetic syndromes, or known chromosomal abnormality. All prospectively enrolled subjects were reevaluated 1 year later to confirm that they remained free of any recognizable systemic disorder, including hypertension. All patients enrolled in the prospective study routinely had echocardiograms performed per the Prematurity and Respiratory Outcomes Program study protocol [17].

For retrospective and prospective groups any subject with an initial echocardiographic reading of moderate or large patent ductus arteriosus (PDA) or atrial level shunt was excluded without review. If the initial reading of shunt size was small to moderate, a senior echocardiographer (MCJ) reviewed the study to exclude any with moderate or larger shunts. PDA was graded as small if the ratio of the smallest ductal diameter to ostium of the left pulmonary artery was $<0.5$ [18]. Atrial shunts were qualitatively graded as small if there was no right ventricular or right atrial enlargement and the color flow Doppler diameter of the shunt was less than $20 \%$ of the length of the atrial septum. Patients with moderate or large shunts on a prior echocardiogram were eligible for inclusion if at least 1 month elapsed until the time of the study echocardiogram.

\section{Echocardiography}

Measurements were made by two-dimensional guided Mmode echocardiography as detailed in a prior study that established normative values [16]. RWT was calculated as twice the left ventricular posterior wall thickness in diastole (LVPWd) over left ventricular end diastolic dimensions (LVEDD)[8, 19]. We selected RWT determined with twice the LVPWd rather than a sum of LVPWd plus interventricular septal dimension given potential interference by tricuspid valve tissue, and right ventricular trabeculations with septal measurement [20, 21]. Thereafter, LV geometry was divided into four categories: normal (left ventricular mass indexed to weight (LVMI) and RWT $<80$ th centile); CR (normal LVMI and RWT $\geq 80$ th centile); CH (LVMI and RWT $\geq 80$ th centile); and EH (LVMI $\geq 80$ th centile and normal RWT). An additional analysis was performed for all patients with elevated RWT (combines $\mathrm{CR}+\mathrm{CH}$ ). Altered LV geometry included patients (Cases) with $\mathrm{CR}, \mathrm{CH}, \mathrm{EH}$, or elevated RWT $(\mathrm{CR}+\mathrm{CH})$. Controls $(n=90)$ were a subgroup of preterm infants who were prospectively enrolled through the Prematurity and Respiratory Outcomes Program with normal LV geometry.

We compared possible risk factors (maternal diabetes, postnatal steroids, drug-treated hypertension, bronchopulmonary dysplasia (BPD), inotrope use, high-frequency oscillator ventilator (HFOV), and small for gestational age (SGA)), and outcomes (duration of hospital stay, fate of altered LV geometry (resolved or not) on follow-up echocardiogram if available, and all-cause mortality) between 
Table 1 Baseline characteristics by left ventricular geometry

\begin{tabular}{lllll}
\hline & $\begin{array}{l}\text { Normal geometry }(n=323, \\
64.2 \%)\end{array}$ & $\begin{array}{l}\text { Concentric remodeling }(n=78, \\
15.5 \%)\end{array}$ & $\begin{array}{l}\text { Eccentric hypertrophy }(n=86, \\
17.1 \%)\end{array}$ & $\begin{array}{l}\text { Concentric hypertrophy }(n=16, \\
3.2 \%)\end{array}$ \\
\hline Males, $n(\%)$ & $158(48.9)$ & $37(47.4)$ & $44(51.2)$ & $9(56.2)$ \\
PMA, weeks & $30.4 \pm 3.9$ & $29.1 \pm 3.6$ & $30.9 \pm 4.0$ & $29.7 \pm 4.0$ \\
Weight, kg & $1.26 \pm 0.44$ & $1.04 \pm 0.40$ & $1.26 \pm 0.45$ & $1.16 \pm 0.42$ \\
LVMI, g/kg & $3.29 \pm 0.75$ & $3.37 \pm 1.30$ & $4.46 \pm 0.46$ & $4.53 \pm 0.48$ \\
RWT & $0.33(0.26-0.38)$ & $0.48(0.43-0.52)$ & $0.32(0.26-0.36)$ & $0.52(0.43-0.60)$ \\
\hline
\end{tabular}

PMA is defined as the time elapsed between the first day of the last menstrual period and birth (gestational age) plus the time elapsed after birth (chronological age); Weight is in $\mathrm{kg}$ on the day of scan; LVMI is left ventricular mass index to weight on day of scan; RWT = the ratio of twice LVPWd over LVEDD. Values are expressed as $n(\%)$ or mean \pm SD as appropriate for all variables except relative wall thickness mean (10th and 80th percentile (P10 and P80))

$P M A$ postmenstrual age, $L V M I$ left ventricular mass index, $R W T$ relative wall thickness, $L V P W d$ thickness of posterior wall of the left ventricle at end diastole, $L V E D D$ left ventricular dimension at end diastole

the case vs. control groups. Serial echocardiographic assessments at staged intervals were not performed in this retrospective study. Follow-up echocardiograms were performed based on clinical concerns.

\section{Statistical analysis}

Continuous variables were summarized as mean $\pm \mathrm{SD}$ (range), (10th and 80th percentile (P10 and P80)) or median (interquartile range (IQR)) as appropriate. Comparisons were performed using independent-samples $t$-test or Mann-Whitney $U$-test or within groups using Wilcoxon signed rank test with Bonferroni correction, as applicable. Categorical variables were presented by the absolute and relative frequencies or as numbers and percentages. Categorical data were compared using the chi-square or the Fisher exact test, as appropriate. Sex-specific LVM indexed for weight centile curve was generated using Cole's lambdamu-sigma estimation method as described previously $[16,22]$. Univariate and multivariate logistic regression with backward selection model were used to identify risk factors associated with all three patterns of altered LV geometry (CR, CH, EH) and for those with elevated RWT (combines $\mathrm{CR}+\mathrm{CH}$ patterns) vs. the control group. Univariate regressions models with $P<0.1$ were included in the multivariate analysis. All statistical tests were two sided, and a $P$-value $<0.05$ was considered statistically significant. Statistical analyses were performed with SAS 9.4 version (SAS Institute, Inc., Cary, NC).

\section{Results}

\section{Demographics}

The distribution of baseline characteristics for our sample ( $n=503$ preterm infants $\leq 2 \mathrm{~kg}$ ) stratified by LV geometry pattern is shown in Table 1. For those with normal LV geometry the mean \pm SD (range) of LVMI was $3.3 \pm 0.75$ $(1.2-7.4) \mathrm{g} / \mathrm{kg}$ and RWT was $0.33 \pm 0.05(0.2-0.41)$.

\section{Altered LV geometry}

Based on 80th centile cut-off values, 180 patients were defined as having altered LV geometry. The patterns of concentric remodeling and eccentric hypertrophy were equally prevalent ( 78 and 86 , respectively), whereas only 16 had concentric hypertrophy (Table 1). Elevated RWT was found in 94 cases.

\section{Risk factors}

With univariate analysis (Table 2) all of the possible risk factors were associated with at least one of the patterns of altered LV geometry. With multiple logistic regression postnatal steroids $(P<0.01$, for all) and SGA (for $\mathrm{CH}$, $P=0.02$ and for $\mathrm{EH}, P=0.04)$ remained as predictors of altered LV geometry (Table 3).

\section{Outcome}

The duration of hospital stay was longer in the elevated RWT (combined $\mathrm{CR}+\mathrm{CH}$ ) cases compared to controls $(P<0.05$ for all, Table 4$)$. Altered LV geometry resolved in all but 2 of the 131 cases with follow-up echocardiograms over a median (IQR) follow-up period of 3 weeks (1.5-6.5). Two patients who had $\mathrm{CR}$ previously progressed to $\mathrm{CH}$ over a period of 1 and 5 weeks prior to death. Nineteen patients with altered LV geometry died compared to 3 in the control group. Mortality was significantly increased in patients with $\mathrm{CR}$ and $\mathrm{CH}$, which includes cases with elevated RWT (Table 4). In the patients with altered geometry who died $(n=19)$, median (IQR) gestational age, birth weight, weight on the day of scan, and age at death were 25 (24-26.5) weeks; 0.7 (0.61-0.79) kg; 0.7 (0.61-0.9) kg; and 29 (10-54) days, respectively. The causes of death in those 
Table 2 Univariate risk factors associated with patterns of altered left ventricular geometry

\begin{tabular}{|c|c|c|}
\hline Risk factors & $\begin{array}{l}\text { Cases/ } \\
\text { controls }\end{array}$ & $\begin{array}{l}\text { Univariate odds ratio }(95 \% \\
\mathrm{CI}) ; P \text {-value }\end{array}$ \\
\hline \multicolumn{3}{|c|}{ Concentric remodeling $(n=78)$} \\
\hline Maternal diabetes & $6: 4$ & $1.3(0.8-2.2) ; 0.5$ \\
\hline Postnatal steroid use & $38: 24$ & $1.6(1.2-2.2) ; 0.004$ \\
\hline Hypertension & $6: 0$ & $2.2(1.9-2.7) ; 0.01$ \\
\hline BPD & $47: 38$ & $1.5(1.1-2.1) ; 0.02$ \\
\hline Inotrope use & $32: 22$ & $1.5(1.1-2) ; 0.02$ \\
\hline HFOV & $33: 27$ & $1.3(0.96-1.8) ; 0.1$ \\
\hline SGA & $6: 4$ & $1.3(0.8-2.2) ; 0.5$ \\
\hline \multicolumn{3}{|c|}{ Concentric hypertrophy $(n=16)$} \\
\hline Maternal diabetes & $3: 4$ & $3.3(1.2-8.8) ; 0.07$ \\
\hline Postnatal steroid use & $12: 24$ & $5.8(2-16.8) ; 0.0002$ \\
\hline Hypertension & $4: 0$ & $8.5(5-14.5) ; 0.0004$ \\
\hline BPD & $11: 38$ & $2.5(1-6.9) ; 0.05$ \\
\hline Inotrope use & $10: 22$ & 3.8 (1.5-9.7); 0.002 \\
\hline HFOV & $10: 27$ & $3.1(1.2-7.9) ; 0.02$ \\
\hline SGA & $4: 4$ & $4.1(1.7-9.8) ; 0.02$ \\
\hline \multicolumn{3}{|c|}{ Eccentric hypertrophy $(n=86)$} \\
\hline Maternal diabetes & $7: 4$ & $1.3(0.8-2.1) ; 0.4$ \\
\hline Postnatal steroid use & $45: 24$ & 1.7 (1.3-2.3); 0.0006 \\
\hline Hypertension & $2: 0$ & $2.1(1.8-2.4) ; 0.2$ \\
\hline BPD & $49: 38$ & $1.3(1-1.8) ; 0.05$ \\
\hline Inotrope use & $31: 22$ & $1.3(1.0-1.8) ; 0.09$ \\
\hline HFOV & $33: 27$ & $1.2(0.9-1.6) ; 0.2$ \\
\hline SGA & $11: 4$ & $1.6(1.1-2.2) ; 0.06$ \\
\hline \multicolumn{3}{|l|}{ Elevated RWT $(n=94)$} \\
\hline Maternal diabetes & $9: 4$ & $1.4(0.9-2.1) ; 0.2$ \\
\hline Postnatal steroid use & $50: 24$ & 1.7 (1.3-2.2); 0.0003 \\
\hline Hypertension & 10:0 & $2.1(1.8-2.4) ; 0.001$ \\
\hline BPD & $58: 38$ & 1.5 (1.1-2); 0.006 \\
\hline Inotrope use & $42: 22$ & $1.5(1.1-2) ; 0.004$ \\
\hline HFOV & $43: 27$ & $1.4(1-1.8) ; 0.03$ \\
\hline SGA & $10: 4$ & $1.4(1-2.1) ; 0.1$ \\
\hline
\end{tabular}

Controls $(n=90)$

$C R$ concentric remodeling, $\mathrm{CH}$ concentric hypertrophy, $E H$ eccentric hypertrophy, $R W T$ relative wall thickness, $B P D$ bronchopulmonary dysplasia, HFOV high-frequency oscillator ventilator, Hypertension drug-treated hypertension, $S G A$ small for gestational age

with altered LV geometry were respiratory failure (6), cardiorespiratory failure (2), necrotizing enterocolitis (7), and sepsis (4). Three patients in the control group died. Their median (IQR) gestational age were 25 (24-26.5) weeks, birth weight $0.78(0.6-0.89) \mathrm{kg}$, weight on the day of scan $0.85(0.78-0.89) \mathrm{kg}$, and age at death were $23(16-33)$ days, respectively. The causes for death in the control group were respiratory failure (1), necrotizing enterocolitis (1), and sepsis (1).

\section{Discussion}

The primary finding in this study of preterm infants is that postnatal steroid use and SGA are risk factors for the development of altered LV geometry. In regards to outcomes, elevated RWT was associated with prolonged hospital stay and increased mortality. This study is the first to provide prognostic significance of altered LV geometry in preterm infants. These data validate previous small studies that show an association between postnatal steroid use and LVH in preterm infants $[11,14]$. Consistent with our data, Skelton et al. [14] found increased risk of transient $\mathrm{LVH}$ following a short-term course of postnatal steroids in preterm infants. Unlike most previous infant studies that focused on single risk factor (e.g., steroids use in preterm infants with bronchopulmonary dysplasia or hypertension), this retrospective case-control study was designed to compare the impact of various risk factors associated with development of altered LV geometry [11-14].

The association of mortality with elevated RWT suggest that this measure provides additional prognostic information beyond that relayed by LVM index alone. This finding is in keeping with the widespread use of RWT in large adult studies [23]. In children, Bharucha et al. [24] reported higher posterior wall $Z$-score as a risk factor for sudden death in patients with hypertrophic cardiomyopathy. The utility of RWT as an outcome predictor could be in part because it does not require indexing to body size. This association does not indicate that cardiac disease was the cause of mortality. Cardiac failure was considered to be a factor in the death for only 2 of 19 patients with altered LV geometry.

Our finding of SGA as a risk factor in the $\mathrm{CH}$ groups could be in part secondary to the use of weight for indexing LVM as supported by the lack of association between RWT and SGA. Conflicting results are found in prior studies comparing indexed LVM in AGA vs. SGA infants [25, 26]. Cinar et al. [25] used body surface area indexing and found decreased indexed LVM in term SGA infants. Although Czernik et al. [26] found equivalent LVM in SGA preterm infants with weight or length indexing, they concluded length is the appropriate index based on indexing results of LV dimension. The assumption that indexed cardiac measures in SGA infants should not be altered potentially masks important cardiac pathology in these infants. Czernik et al. [26] did point out the practical difficulty of the tape measure length method in preterm infants.

We selected the formula for RWT that excludes septal thickness for pattern classification given interference with septal measurement from tricuspid valve tissue and right ventricular trabeculations. Previous studies acknowledged similar issues with inaccurate M-mode echocardiographic diagnosis of asymmetric septal hypertrophy due to 
Table 3 Multivariate risk factors associated with patterns of altered left ventricular geometry

\begin{tabular}{lllll}
\hline Risk factors & CR & CH & EH & Elevated RWT \\
$(N=78)$ & $(N=16)$ & NS & NS \\
\hline Maternal diabetes & NS & NS & $3.2(1.67-6.05) ;$ & $3.1(1.6-5.7) ;$ \\
Postnatal steroid & $2.5(1.33-4.86) ;$ & $8.3(2.33-29.90) ;$ & 0.0004 & NS \\
use & 0.005 & 0.001 & NS & NS \\
Hypertension & NS & NS & NS & NS \\
BPD & NS & NS & NS & NS \\
Inotrope use & NS & NS & NS & NS \\
HFOV & NS & NS & $3.7(1.08-12.42) ;$ & 0.04 \\
SGA & NS & $7.3(1.30-41.38) ;$ & \\
\hline
\end{tabular}

Controls $(n=90)$. Values are expressed as odds ratio $(95 \% \mathrm{CI}) ; P$-value

$C R$ concentric remodeling, $C H$ concentric hypertrophy, $E H$ eccentric hypertrophy, RWT, relative wall thickness, Hypertension drug-treated hypertension, $S G A$ small for gestational age

interference by normal right heart structures [20, 21]. It is possible that RWT, which includes septal thickness, would have a closer association with maternal diabetes given the predilection for septal hypertrophy in these infants [27-29]. In a prospective fetal study, Cooper et al. [29] found a $31 \%$ prevalence of septal hypertrophy in mothers with diabetes starting at 31-34 weeks of gestation. A follow-up study comprised of mostly term infants of diabetic mothers showed resolution of hypertrophy by 6 months [30]. The lack of association of altered LV geometry and maternal diabetes in our study may, in part, be secondary to prematurity. Further prospective studies are needed to assess the potential impact of maternal diabetes on cardiac morbidity in preterm infants.

Most of our cases with increased RWT fall into the CR group. Large adult studies find that $\mathrm{CR}$ is an early response to pressure overload, whereas $\mathrm{CH}$ occurs later and is associated with the most adverse outcome [23]. Serial studies with attention to blood pressure measurements are needed to test the applicability of this concept in preterm infants. With multivariate analysis postnatal steroid use was a predictor in all patterns of altered LV geometry. Prospective trials would be necessary to determine if steroids had an impact on morbidity or mortality.

With improving survival of extremely low birth weight infants, the prevalence of concentric and eccentric hypertrophy may increase. These findings may influence risk assessment and impact decision making of clinicians caring for these high-risk infants. Typically, 95th percentile is used to define pathology in 'healthy' pediatric populations. Similar to Lieb et al. [8] study in adults we used an 80th centile cut-off for relative wall thickness and indexed LVM for defining altered LV geometry. This lower threshold was chosen in order to capture LV pathology in a hospitalized preterm cohort expected to have a high prevalence of disease.

\section{Limitations}

The retrospective nature of our multivariate analysis may include unmeasured confounders. Our study population is likely biased towards a higher prevalence of abnormal LV geometry because most of these patients underwent echocardiography for clinical reasons and did not represent a consecutive birth cohort. Additionally, selection bias for worse disease is likely in these infants cared for at a tertiary care referral center. The high incidence of PDA in the preterm population makes it difficult to eliminate PDA as a confounding factor. These infants may have had persistent altered LV dimensions from a moderate to large PDA present at some time prior to the study echocardiogram. Although our results are not applicable to infants with pulmonary hypertension as this finding was an exclusion factor, compression of the LV by a hypertensive right ventricle could lead to falsely elevated RWT and decreased LVMI. Concentric hypertrophy group comprised only $3 \%$ of the study population. The low statistical power may have undermined the chance of detecting a true effect. Also, details on the duration of postnatal steroid use, severity, and duration of hypertension that would be expected to affect the evolution of LVH were not evaluated. Over $25 \%$ of our cases did not have a follow-up echocardiogram, either because they died or were transferred back to their referring hospital or there were no clinical concerns necessitating follow-up echocardiogram. Follow-up data on resolution of altered LV geometry need to be interpreted with caution because serial echocardiographic assessments at staged intervals were not performed. We used M-mode echocardiography for quantitative estimation of LVM and RWT given the ease of use in a clinical setting [16]. MRI imaging would be an ideal validation tool but has practical challenges in this population. Although our data show an association of mortality with altered LV geometry, we 
Table 4 Outcomes associated with patterns of altered left ventricular geometry

\begin{tabular}{lll}
\hline & Hospital stay & Mortality \\
\hline $\mathrm{CR}$ & $101 \pm 41$ vs. $94 \pm 27, P=0.1$ & $12 / 78$ vs. 3/90, OR 2.8, 95\% CI $(1.02-7.90), P=0.006$ \\
$\mathrm{CH}$ & $141 \pm 94$ vs. $94 \pm 27, P=0.007$ & $3 / 16$ vs. 3/90, OR $1.7,95 \% \mathrm{CI}(0.78-3.89), P=0.04$ \\
$\mathrm{EH}$ & $104 \pm 41$ vs. $94 \pm 27, P=0.06$ & $4 / 86$ vs. 3/90, $P=0.6$ \\
Elevated RWT $(\mathrm{CR}+\mathrm{CH})$ & $107 \pm 55$ vs. $94 \pm 27, P=0.04$ & $15 / 94$ vs. 3/90, OR 3.1, 95\% CI $(1.1-8.9), P=0.004$ \\
\hline
\end{tabular}

Controls $(n=90)$. Values are expressed as mean \pm SD for the above hospital stays for patients who survived

$C R$ concentric remodeling, $\mathrm{CH}$ concentric hypertrophy, $E H$ eccentric hypertrophy, $R W T$ relative wall thickness, $O R$ odds ratio, $S G A$ small for gestational age

cannot conclude that cardiac disease is a cause of death in these patients. Further studies, preferably prospective, are needed to corroborate our findings, and uncover additional links between preterm morbidities and cardiac hypertrophy.

\section{Conclusions}

This study determined that altered LV geometry in preterm infants is related to postnatal steroid use and SGA. Although altered LV geometry often resolves, it is associated with a poor outcome in preterm infants with a longer duration of hospital stay and higher mortality. RWT does not involve indexing to body dimensions and is a potentially important tool for studying cardiac pathology in preterm infants.

Author contributions MCJ and SC conceptualized and designed the study, drafted the initial manuscript, critically reviewed, revised, and approved the final manuscript as submitted. AS carried out the statistical analyses and critically reviewed and revised the manuscript. TWC collected data and critically reviewed the manuscript. PTL, $\mathrm{BPH}$, and GKS designed the data collection and critically reviewed and revised the manuscript. All authors approved the final manuscript as submitted and agree to be accountable for all aspects of the work.

Funding This work was supported in part by a grant from the Premature and Respiratory Outcomes Program (PROP) National Institutes of Health (NIH) (grant U01 HL101794 and HL1014650).

\section{Compliance with ethical standards}

Conflict of interest The authors declare that they have no conflict of interest.

\section{References}

1. Casale PN, Devereux RB, Milner M, Zullo G, Harshfield GA, Pickering TG, et al. Value of echocardiographic left ventricular mass in predicting cardiovascular morbid events in hypertensive men. Ann Intern Med. 1986;105:173-8.

2. Levy D, Garrison RJ, Savage DD, Kannel WB, Castelli WP. Prognostic implications of echocardiographically determined left ventricular mass in the Framingham Heart Study. N Engl J Med. 1990;322:1561-6.
3. Foster BJ, Mackie AS, Mitsnefes M, Ali H, Mamber S, Colan SD. A novel method of expressing left ventricular mass relative to body size in children. Circulation. 2008;117:2769-75.

4. Hanevold C, Waller J, Daniels S, Portman R, Sorof J. International Pediatric Hypertension Association. The effects of obesity, gender, and ethnic group on left ventricular hypertrophy and geometry in hypertensive children: a collaborative study of the International Pediatric Hypertension Association. Pediatrics. 2004;113:328-33.

5. Urbina EM, Gidding SS, Bao W, Pickoff AS, Berdusis K, Berenson GS. Effect of body size, ponderosity, and blood pressure on left ventricular grow thin children and young adults in the Bogalusa Heart Study. Circulation. 1995;91:2400-6.

6. De Simone G, Daniels SR, Devereux RB, Meyer RA, Roman MJ, de Divitiis O, et al. Left ventricular mass and body size in normotensive children and adults: assessment of allometric relations and impact of overweight. J Am Coll Cardiol. 1992;20:1251-60.

7. Milani RV, Lavie CJ, Mehra MR, Ventura HO, Kurtz JD, Messerli $\mathrm{FH}$. Left ventricular geometry and survival in patients with normal left ventricular ejection fraction. Am J Cardiol. 2006;97:959-63.

8. Lieb W, Gona P, Larson MG, Aragam J, Zile MR, Cheng S, et al. The natural history of left ventricular geometry in the community: clinical correlates and prognostic significance of change in LV geometric pattern. JACC Cardiovasc Imaging. 2014;7:870-8.

9. Lavie CJ, Milani RV, Ventura HO, Cardenas GA, Mehra MR, Messerli FH. Disparate effects of left ventricular geometry and obesity on mortality in patients with preserved left ventricular ejection fraction. Am J Cardiol. 2007;100:1460-4.

10. Aye CYL, Lewandowski AJ, Lamata P, Upton R, Davis R, Ohuma EO, et al. Disproportionate cardiac hypertrophy during early postnatal development in infants born preterm. Pediatr Res.2017;82:36-46.

11. Dani C, Bertini G, Simone P, Rubaltelli FF. Hypertrophic cardiomyopathy in preterm infants treated with methylprednisolone for bronchopulmonary dysplasia. Pediatrics. 2006;117:1866-7.

12. Wang KC, Botting KJ, Padhee M. Early origins of heart disease: low birth weight and the role of the insulin-like growth factor system in cardiac hypertrophy. Clin Exp Pharmacol Physiol. 2012;39:958-64.

13. Peterson AL, Frommelt PC, Mussatto K. Presentation and echocardiographic markers of neonatal hypertensive cardiomyopathy. Pediatrics. 2006;118:782-5.

14. Skelton R, Gill AB, Parsons JM. Cardiac effects of short course dexamethasone in preterm infants. Arch Dis Child Fetal Neonatal Ed. 1998;78:F133-F137.

15. Foster BJ, Khoury PR, Kimball TR, Mackie AS, Mitsnefes M. New reference centiles for left ventricular mass relative to lean body mass in children. J Am Soc Echocardiogr. 2016;29:441-7.

16. Choudhry S, Salter A, Cunningham TW, Levy PT, Nguyen HH, Wallendorf $M$. et al. Normative left ventricular M-mode echocardiographic values in preterm infants up to two kilograms. J Am Soc Echocardiogr. 2017;30:781-9.e4. 
17. Levy PT, Dioneda B, Holland MR, Sekarski TJ, Lee CK, Mathur A, et al. Right ventricular function in preterm and term neonates: reference values for right ventricle areas and fractional area of change. J Am Soc Echocardiogr. 2015;28:559-69.

18. Ramos FG, Rosenfeld CR, Roy L, Koch J, Ramaciotti C. Echocardiographic predictors of symptomatic patent ductus arteriosus in extremely-low-birth-weight preterm neonates. J Perinatol. 2010;30:535-9.

19. Biton Y, Goldenberg I, Kutyifa V, Baman JR, Solomon S, Moss AJ, et al. Relative wall thickness and the risk for ventricular tachyarrhythmias in patients with left ventricular dysfunction. Am Coll Cardiol. 2016;67:303-12.

20. Bernstein RF, Tei C, Child JS, Shah PM. Angled interventricular septum on echocardiography: anatomic anomaly or technical artifact? J Am Coll Cardiol. 1983;2:297-304.

21. Keren A, Billingham ME, Popp RL. Echocardiographic recognition of paraseptal structures. J Am Coil CardioI. 1985;6:913-9.

22. Cole TJ, Green PJ. Smoothing reference centile curves: the LMS method and penalized likelihood. Stat Med. 1992;11:1305-19.

23. Gaasch WH, Zile MR. Left ventricular structural remodeling in health and disease: with special emphasis on volume, mass, and geometry. J Am Coll Cardiol. 2011;58:1733-40.

24. Bharucha T, Lee KJ, Daubeney PE, Nugent AW, Turner C, Sholler GF, et al. Sudden death in childhood cardiomyopathy: results from a long-term national population-based study. J Am Coll Cardiol. 2015;65:2302-10.

25. Cinar B, Sert A, Gokmen Z, Aypar E, Aslan E, Odabas D. Left ventricular dimensions, systolic functions, and mass in term neonates with symmetric and asymmetric intrauterine growth restriction. Cardiol Young-. 2015;25:301-7.

26. Czernik C, Rhode S, Metze B. Comparison of left ventricular cardiac dimensions between small and appropriate for gestational age preterm infants below 30 weeks of gestation. J Perinat Med. 2013;41:219-226.

27. Ullmo S, Vial Y, Di Bernardo S, Roth-Kleiner M, Mivelaz Y, Sekarski N, et al. Pathologic ventricular hypertrophy in the offspring of diabetic mothers: a retrospective study. Eur Heart J. 2007;28:1319-25.

28. Haşmaşanu MG, Bolboaca SD, Matyas M, Zaharie GC. Clinical and echocardiographic findings in newborns of diabetic mothers. Acta Clin Croat. 2015;54:458-66.

29. Cooper MJ, Enderlein MA, Tarnoff H, Rogé CL. Asymmetric septal hypertrophy in infants of diabetic mothers. Fetal echocardiography and the impact of maternal diabetic control. Am J Dis Child. 1992;146:226-9.

30. Gutgesell HP, Speer ME, Rosenberg HS. Characterization of the cardiomyopathy in infants of diabetic mothers. Circulation. 1980;61:441-50. 